\title{
The Translation of Tourism Materials of Scenic Spots from the Perspective of Nida's Functional Equivalence Theory
}

\author{
Ling Wang \\ School of foreign languages, Nanchang Normal University, Nanchang, China
}

1151401144@qq.com

\begin{abstract}
Keywords: Tourism material; Functional equivalence theory; Current situation in China; Tourism material translation
\end{abstract}

\begin{abstract}
With the growth of China's economy and its thriving tourism industry, the quantity of foreign visitors coming to China has a huge growth. Since the scenic-spot introduction performs as the bridge between scenic spots and tourists, the translation quality plays an important part in foreign tourism. Firstly, this thesis states the main content of Nida's functional equivalence theory, then points out several specific problems in the current situation of the tourism material translation in China; Finally, It explores how to integrate the functional equivalence theory with practical translating process and puts forward some corresponding suggestions of applying functional equivalence theory into tourism material translation.
\end{abstract}

\section{Introduction}

With the enhancement of the people's living quality, the tourism has become an indispensable daily activity for more and more people. As one of the most popular ways for people to relax, tourism also is the key to the cultural communication between different cultures. Under such a circumstance, tourism materials translation is of great importance in terms of conveying cultural message, and it is quite necessary to find a proper way to produce high-quality translation and it goes according to the following steps:

Actually, there is a contradiction between the supply and demand. The need for tourism materials translation is on the rise while the study in this field is somewhat neglected and the quality of its translation is usually undesirable. Some researchers consider that Nida's functional equivalence theory can effectively guide the tourism material translation as it aims to get "the closest natural equivalence" in translating. This paper attempts to reveal the possibility of applying functional equivalence theory into tourism material translation. [1]

Firstly, the knowledge of tourism material is examined to help evaluate if the theory is suitable to guide tourism material translation. Due to the categorization and the main features of tourism material, it is impossible to make a "correct" translation to cater the needs of the readers. Actually, Compared with any other elements such as form and style, the translating of meaning is more important.

Secondly, there are several concrete issues that a translator would encounter in tourism material translation. It is quite conductive to discuss the barriers and proposing useful techniques, which provide a method to use the functional equivalence theory into the tourism material translation.

Thirdly, after having done the theoretical review, it is necessary to do some instance studies. Compared English culture with Chinese culture and their translated versions at different levels, the techniques to realize functional equivalence theory in tourism material translation are clarified in the light of functional equivalence theory. [2]

From the above, it is obvious that it can conclude that functional equivalence theory is applicable to tourism material translation. In a word, this paper discusses the strategies of how to get high-quality tourism material translation in an effective way. The translated version of tourism material should be in conformity with the target language, culture, customs, etc. Thus the translator should take these factors into consideration in practical translation.

This thesis studies English and Chinese and their translated versions, trying to distinguish tourism 
material in these two languages. The author aims to find an approach to produce high-quality tourism material translations in an effective way.

\section{Important Concepts in Nida's Functional Equivalence Theory.}

Eugene A. Nida is a famed and outstanding American translation theorists. He has made scientific researches in many fields including linguistics, semantics, anthropology, etc. He is one of the most productive translation theorists and his works have great influence in translation field.

As a researcher says "Nida devoted himself to the development of translation studies. He has worked through his lifetime in helping people break "the Tower of Babel". Many scholars have triggered a heated discussion as his functional equivalence theory come up, the functional equivalence has been a significant counseling theory to the translation. [3]

Correspondence. In translation research, especially when analyzes functional equivalence, there is a word that should not be ignored-correspondence. In his book Toward a Science of Translating, he uses "correspondence" to denote a broad concept which covers both formal and dynamic equivalence. For him, this word represents the relationship not only between source language and target language symbols, but also between the ways such units are arranged within the structures of the two languages. [4]

There are suggestions from Nida, showing how to get the satisfactory correspondence. Generally speaking, three lexical levels should be considered: Terms for which can be actually correspond translated, e.g. river, sky, building, temple etc.; Terms which identify culturally different objects, but with somewhat analogous functions, e.g. the inner implication of dog in English and Chinese (in English, dog is usually used to express loyalty or commendatory term, because western people regard dogs as their best friends: love him love his dog. But in Chinese, dog is always related with derogatory term: “狐朋狗友”, as Chinese regard dog as lowliness); Terms which identify cultural specialties, e.g. Christmas in English is what the spring festival in Chinese. [5]

Besides, if source and receptor languages represent very different cultures there should be many basic themes and accounts which cannot be "naturalized" by the process of translating. Since tourism is closely related to the present times other than the past one, translators should deal with the second type more carefully to avoid errors.

Formal Equivalence. Formal equivalence revolves around both the form and content of the message. As formal equivalence focuses on oriented text in both form and content, it can be regarded as source-oriented as it is designed to get as close as possible of the original version. [6]

According to formal translation, the receptor is required in a sense to learn or understand the convention, etiquette, ways of presentation, and the other elements of the culture that may take place in the source language. In addition, that means he may at least have the ability to read as well as an average receptor from the source language environment. Otherwise, the author must make a deeper interpretation as a supplement for the translation. So the receptors can fully understand what the author writes.

It is obvious that formal equivalence translation is appropriate in certain circumstance, offering some extent of discernment to the source text. In other words, the relative value and effectiveness of particular types of translations for particular receptors must not be confused with the description of the nature of various kinds of translations.

Dynamic Equivalence. The focus of Dynamic equivalence is the dynamic relationship; Dynamic equivalence doesn't need translators to make the target-language and the source-language consistent. [7]

A dynamic equivalent translation does not require the receptor to understand the patterns of the source language culture. In fact, it tries to relate the receptor the way of thought or behavior with his own culture to natural expressions. In this point, this kind of translation focuses not so much on the source message but on the reader's response.

And Nida also proposes a way to achieve dynamic equivalence: Translation is a process to make the target language achieve the proximate natural equivalent to the message of the source language. 
The first is in terms of meaning and secondly is in terms of style."

Functional Equivalence in Tourism Material Translation. Apparently, equivalence should not be considered as sameness. It is easy to see that there is no word in one language can be found having precisely the same meaning of another word in another language. Of course, here the "meaning" refers not only the connotation of a word, but also its denotation.

All in all, according to Nida's theories, effective tourism material translations can be achieved under the guidance of functional equivalence theory. To attain the "closet natural equivalence", we need to consider many factors. And how this theory is realized in practical tourism material translation will be discussed in the following chapters.[8]

\section{Problems Existing in the Tourism Materials Translation}

There are several problems still existing in the tourism material translation of scenic spots of China. The tourism material can't only provide detailed information but also attract more tourists. English version of tourism material of Chinese scenic spots should achieve the same aims, because it is the translated versions of the tourism material that have become the critical approaches to disseminate Chinese culture to the world and accelerate the development of Chinese tourism industry.

Cultural Mistranslation. Due to the discrepancies of the historical development between China and western countries, their culture, such as literature, history, geography, philosophy, science and technology, industry and agriculture are also distinct. So, translators usually make this kind of mistakes. For example:

In the mind of Chinese people, "Dragon" is the legendary "God", it is deemed to the symbol of right or power, its cultural meaning is "noble, sacred and rare." On the contrary, in the western culture, the dragon always refers to a huge lizard which dragging a long tail, and the whole body covered with scales, whose mouth can Spit fire. Thus it is the symbol of "evil", and its cultural meaning is "monster, devil".

Misnomer. Chinese words can't fully correspond with the English words, so maybe a Chinese word can be translated into several English words. Just as the Chinese verb “打”, which is most commonly used in Chinese, but has many different meaning, such as make a call, have a nap, fetch water. So when we translate such words we should choose the most appropriate words. [9]

In China, people would like to quote a lot of ancient prose into the tourism materials to improve the cultural connotation of the scenic spots. However, the meaning of some terms or phrases in the past is quite diverse from now. So it's difficult to translate these tourism materials with both the national features and the meaning of the original.

\section{Techniques in Tourism Material Translation}

According to Nida, what a translator really should do is to select in each case the most appropriate natural equivalent.

The aims of translation techniques are stated by Nida as: permit modulation of the form of the text to the demands of the structure of the target language; produce semantically equivalent structures; provide equivalent stylistic appropriateness; carry an equivalent communication load.

In order to reach the above aims, there are two things for the translator to bear in mind. Firstly, the basic task for him is to reproduce what he has been given, not to improve it. Secondly, what kind of adjustment should be made depends largely on the receptor for which the translation is designed.

As what have been discussed above, the translation techniques have such following ways: amplification, omission and rearrangement of information unite. Amplification: Completing information of the original text is a major way of amplification. It can be realized by either adding complementary to the main body or giving footnotes. Omission: Omitting or deleting certain information is often necessary in translating. [10] as we have discussed before, different languages or cultures have their own ways to express. Then making some adjustments is reasonable to make 
the translated text acceptable and fluent in the target language. Rearrangement of Information Unites: As a matter of fact, a satisfactory translation cannot be done with just adding or deleting some words or phrases. Rearranging the information unites is the only way to reach the closest natural equivalence between the source and target text. Different from literature translation, tourism material translation has its unique function and therefore has its own way to be translated. As we know, the primary function of tourism material is to transform information and culture. So, if the translated text can do the same work as the original tourism material does, it can be regarded as a qualified one.

\section{Suggestions for the Tourism Materials Translation}

Being different from other traditional translation theories, Nida's functional equivalence theory authorizes a translator to have more freedom to deal with problems of diversities in types, differences in receptors, and distinctions in purposes that the translation texts aim to achieve, etc. And as the most important point, Nida pay attention to the reader's response, not only limited at verbal comparison level between the source language and the receptor language. [11]

Based on the functional equivalence theory, the primary function of tourism materials is to convey information about tourism resources and to arouse traveling interests in tourists. Due to different cultural regulations, Chinese readers and English readers have different information expectations. Some information may be deemed as successful and sufficient in Chinese but it may be unnecessary in English and even cause difficulties in information conversion. So the information focus in source text should be varied in the view of target readers so that functional equivalence could be achieved between the source text and the target text.

Analogy. Analogy is a ideal technique to solve cultural differences in cross-cultural communication. In the analogy, cultural information in the source text can be converted into something acquainted to the target receptors. It can help the foreign visitors to have a rough understanding of what is in the source text. In this way, even who have little knowledge about Chinese culture can also comprehend the general meaning of the tourism materials. Of course, the premise of using this technique is that there must be certain similarities between the western and Chinese cultural information system. The following example can show the application of this way.

济公劫富济贫，深受穷苦人民爱戴。

Jigong, Robin Hood in China, robbed the rich and helped the poor.

The translator compares Robin Hood with Jigong, so that foreigner can know why he is so respected by Chinese, and then the receptor can have a better and deeper understanding of the sentence. [12]

Restructuring. Restructuring as a translation technique, it means to dissociate from the original structure of the sentences in the source message. Then restructure these sentences in a way is more corresponding to the receptor message. In fact, if the translators use the same sentence structure as its Chinese counterpart, contrary it will produce some sentences which may sound unnatural towards foreigners, and even sometimes the original meaning could be misinterpreted. For example:

“洞庭湖“衔远山，吞长江，浩浩汤汤，横无际涯。朝晖夕阴，气象万千。”[13]

"Carrying distant mountains in the mouth and swallowing the Yangtze River, the vast and mighty Dongting Lake stretches endlessly. It turns brilliant in the morning and gloomy at dust. The scenery abounds in changes."

In the above example, for Chinese receptors, the Chinese edition can be well written by exploiting parallel structures. If translators translate such kind of sentences according to the original Chinese version, it will certainly create a tedious and detailed expression.

\section{Conclusions}

Equivalence is always a key issue to most translation theorists. Nida deals with this concept within his functional equivalence theory in a comprehensive and flexible way, which makes it possible to 
realize this theory in tourism material translation.

There are several problems lying in the transmission of both linguistic and cultural messages. The author chooses English and Chinese tourism material to do the research on practical translation, and attempts to find an effective approach to create high-quality translation in the light of functional equivalence theory. The exploration in this thesis has demonstrated the probability of applying functional equivalence theory to practical tourism material translation.

This study aims not only to provide a way of translating by considering the response of both source and target language receptors, but also to enlighten further research to fulfill the increasing requirements for tourism material translation.

\section{References}

[1] Nida, Eugene A. Toward A Science in Translating [M]. Shanghai: Foreign Language Teaching and Research Press, China 2004. p159.

[2] Nida, Eugene A. Language, Culture and Translating [M]. Shanghai: Foreign Language Teaching and Research Press, China 1993. p12.

[3] Nida, Eugene A. The Theory and Practice of Translation [M]. Shanghai: Shanghai Foreign Language Education Press, China 2004. p32.

[4] Nida, E. A. Language and Culture: Context in Translating [M]. Shanghai: Shanghai Foreign Language Education Press, China, 2001 p57-59.

[5] Hj Ma. A Study on Nida's Translation Theory [M]. Shanghai: Foreign Language Teaching and Research Press, China 2003. p30-31 